\title{
THE DISTANCE BETWEEN THE EIGENVALUES OF HERMITIAN MATRICES
}

\author{
RAJENDRA BHATIA
}

\begin{abstract}
It is shown that the minmax principle of Ky Fan leads to a quick simple derivation of a recent inequality of V. S. Sunder giving a lower bound for the spectral distance between two Hermitian matrices. This brings out a striking parallel between this result and an earlier known upper bound for the spectral distance due to L. Mirsky.
\end{abstract}

Let $A$ be a Hermitian matrix of order $n$ and let $\lambda_{\downarrow}(A)$ denote the vector in $\mathbf{R}^{n}$ whose coordinates are the eigenvalues of $A$ arranged as $\lambda_{[1]}(A) \geq \cdots \geq \lambda_{[n]}(A)$. Let $\lambda_{(1)}(A) \leq \cdots \leq \lambda_{(n)}(A)$ be the increasing rearrangement of these eigenvalues and $\lambda_{\uparrow}(A)$ the vector with coordinates $\lambda_{(j)}(A), j=1,2, \ldots, n$. The same symbols $\lambda_{\downarrow}(A)$ and $\lambda_{\uparrow}(A)$ will also denote the diagonal matrices which have as their diagonal entries the components of the vectors $\lambda_{\downarrow}(A)$ and $\lambda_{\uparrow}(A)$, respectively. Let $\|\cdot\|$ denote any unitarily invariant norm on the space of matrices. (See $[\mathbf{4}]$.)

This note is concerned with the following result:

THEOREM. Let $A$ and $B$ be Hermitian matrices. Then for every unitarily invariant norm we have

$$
\left\|\lambda_{\downarrow}(A)-\lambda_{\downarrow}(B)\right\| \leq\|A-B\| \leq\left\|\lambda_{\downarrow}(A)-\lambda_{\uparrow}(B)\right\| .
$$

The first inequality in (1) appeared in a paper of Mirsky [4], who used a famous result of Lidskii and Wielandt to derive it. The second is proved in a recent paper of Sunder [5]. I give here another proof of the second inequality which has two attractive features: It is very short and it proceeds on exactly the same lines as the well-known proof of Lidskii, Wielandt and Mirsky for the first inequality. For illumination, I indicate how both inequalities follow from the same principle.

It is an easy consequence of the minmax principle of Wielandt that for any choice $1 \leq i_{1}<\cdots<i_{k} \leq n$ of $k$ indices we have

$$
\sum_{j=1}^{k} \lambda_{\left[i_{j}\right]}(A+B) \leq \sum_{j=1}^{k} \lambda_{[j]}(A)+\sum_{j=1}^{k} \lambda_{\left[i_{j}\right]}(B)
$$

for all $k=1,2, \ldots, n$, with equality holding for $k=n$. (See [3, p. 242].)

Writing $x \prec y$ to mean that the vector $x$ is majorised by the vector $y$ in $\mathbf{R}^{n}$ (see $[\mathbf{3}])$, we get from inequalities (2)

$$
\lambda_{\downarrow}(A+B)-\lambda_{\downarrow}(B) \prec \lambda_{\downarrow}(A) .
$$

With a change of variables, this gives

$$
\lambda_{\downarrow}(A)-\lambda_{\downarrow}(B) \prec \lambda_{\downarrow}(A-B) .
$$

Received by the editors August 27, 1984 and, in revised form, January 14, 1985.

1980 Mathematics Subject Classification. Primary 15A42, 15 A57.

Key words and phrases. Hermitian matrices, eigenvalues, minmax principle, majorisation. 
Now the first part of the Theorem follows using standard characterisations of majorisation together with properties of symmetric gauge functions and unitarily invariant norms. This is the well-known proof of Mirsky [4].

Now note that from (2) we can also conclude

$$
\lambda_{\downarrow}(A+B) \prec \lambda_{\downarrow}(A)+\lambda_{\downarrow}(B) .
$$

In fact, for this conclusion the full force of $(2)$ is not needed. It suffices to use the special case $\left(i_{1}, \ldots, i_{k}\right)=(1, \ldots, k)$ which is much easier to prove using the minmax principle of Ky Fan [2].

Replace $B$ by $-B$ in (4) and note that $\lambda_{\downarrow}(-B)=-\lambda_{\uparrow}(B)$. This gives

$$
\lambda_{\downarrow}(A-B) \prec \lambda_{\downarrow}(A)-\lambda_{\uparrow}(B) .
$$

But this implies

$$
\begin{aligned}
\left(\left|\lambda_{[1]}(A-B)\right|, \ldots,\left|\lambda_{[n]}(A-B)\right|\right) & \\
\prec_{\mathrm{w}} & \left(\left|\lambda_{[1]}(A)-\lambda_{(1)}(B)\right|, \ldots,\left|\lambda_{[n]}(A)-\lambda_{(n)}(B)\right|\right)
\end{aligned}
$$

where $\prec_{\mathrm{w}}$ stands for weak majorisation [3, p. 116].

Let $s_{[j]}(A)$ denote the $j$ th singular value of $A$. Let $\|A\|_{k}=s_{[1]}(A)+\cdots+s_{[k]}(A)$ for $k=1,2, \ldots, n$. Then (5) can be restated as $\|A-B\|_{k} \leq\left\|\lambda_{\downarrow}(A)-\lambda_{\uparrow}(B)\right\|_{k}, k=$ $1,2, \ldots, n$. So the second inequality in (1) holds for this special class of norms and hence, by a well-known theorem of Ky Fan, for every unitarily invariant norm. (See $[4]$.

It should be remarked that Sunder's paper contains a stronger result in that it also establishes an analogue of the second inequality in (1) for the case when $A, B$ and $A-B$ are all normal. Under these conditions an analogue of the first inequality in (1) has been established in [1].

\section{REFERENCES}

1. R. Bhatia, Analysis of spectral variation and some inequalities, Trans. Amer. Math. Soc. 272 (1982), 323-331.

2. Ky Fan, On a theorem of Weyl concerning eigenvalues of linear transformations. I, Proc. Nat. Acad. Sci. U.S.A. 35 (1949), 652-655.

3. A. W. Marshall and I. Olkin, Inequalities: Theory of majorisation and its applications, Academic Press, New York, 1979.

4. L. Mirsky, Symmetric gauge functions and unitarily invariant norms, Quart. J. Math. Oxford (2) 11 (1960), 50-59.

5. V. S. Sunder, On permutations, convex hulls and normal operators, Linear Algebra and Appl. 48 (1982), 403-411.

Indian Statistical Institute, 7, SJS SANSANWAL MaRG, NEW DELhi-110016, INDia 